\title{
Comparative Study of Heterostructure Barrier Diodes in the GaAs/AIGaAs System
}

\author{
Mise Akura ${ }^{1, ~ *, ~ G e o f f r e y ~ D u n n ², ~ M o h a m m e d ~ M i s s o u s ~}{ }^{3}$ \\ ${ }^{1}$ Department of Electronic Engineering, University of Aberdeen, Aberdeen, UK \\ ${ }^{2}$ Department of Physics, University of Aberdeen, Aberdeen, UK \\ ${ }^{3}$ Department of Electrical and Electronic Engineering, University of Manchester, Manchester, UK \\ Email address: \\ r01mja16@abdn.ac.uk (M. Akura), g.m.dunn@abdn.ac.uk (G. Dunn) \\ *Corresponding author
}

\section{To cite this article:}

Mise Akura, Geoffrey Dunn, Mohammed Missous. Comparative Study of Heterostructure Barrier Diodes in the GaAs/AlGaAs System. International Journal of Materials Science and Applications. Vol. 7, No. 4, 2018, pp. 161-166. doi: 10.11648/j.ijmsa.20180704.17

Received: August 3, 2018; Accepted: September 12, 2018; Published: September 18, 2018

\begin{abstract}
A comparative study of the electron transport property and operation of the Potential Well Barrier (PWB) diode and Planar-doped Potential-well Barrier (PPB) diode has been carried out in this study. Both diodes are heterostructures in GaAs/AlGaAs system with similarities in layer design though, with a sheet charge inserted close to the well of the PPB diode. A drift-diffusion and Monte Carlo simulation models were used throughout the study to examine the behavior of electrons in terms of the electric field distribution across the diodes, electron velocities, electron energy and densities. Results of simulation has shown how the electric field varies in the left and right intrinsic regions of the device and the effect of the field on velocity. The I-V characteristics of the experimental and simulation results have shown a good agreement in the two diodes though, with little adjustment of about $\pm 2.5 \%$ to design parameters in order to obtain a good fit with experimental results. The I-V characteristics of the diodes reveal that the PPB diode turns on at a higher voltage than the PWB diode though, with a better asymmetry in the reverse bias operation. This is because the sheet charge in the PPB diode produces additional charge and together with the charge in the well, presents a higher potential barrier than the PWB diode whose barrier is determined by the charge in the well only. The diodes demonstrate promising RF behavior with voltage responsivity of $10900 \mathrm{~V} / \mathrm{W}$ and $6400 \mathrm{~V} / \mathrm{W}$ at $10 G H$ for the PPB and PWB diodes respectively.
\end{abstract}

Keywords: Monte Carlo, Drift-Diffusion, Potential Well, Curvature Coefficient, Turn-on Voltage

\section{Introduction}

The operation of the potential well barrier (PWB) and the hybrid planar-doped potential-well (PPB) barrier diodes have been investigated previously using drift-diffusion models [1-4] without any treatment of energy transport or heating effects. Though diodes of this kind such as the Heterostructure Barrier diodes (HBDs) [5] and Heterostructure Barrier Varactors (HBVs) [6] were investigated in the past. The PWBs and PPBs are similar in design to these diodes with majority carriers and potential applications in heterodyne detection and mixers. Just like the PDBs, these diodes have the advantage of a controllable barrier height and also offer some improvements in the asymmetry due to active nature of charge in the well. Thus, considering hot electron effects was important in these devices [7-9]. In this paper, discussion will be on how, whilst the basic semiconductor theory sufficiently describes the behaviour of these diodes, a more advanced treatment of the diode is needed to understand the details of carrier dynamics in the device. The paper will also demonstrate how the electric field, mean energy and velocity of electrons across each diode varies using a hot carrier model.

The I-V characteristics produced from experiments and simulation of the PWB and PPB diodes will be compared. The PWB diode is expected to have a smaller asymmetrical structure compared to the PPB diode and hence a lower turn-on voltage and the paper will show why this is possible. The $d c$ characteristics of the diodes which determines diodes' 
performance in both the forward and reverse bias operations will also be compared.

\section{Experimental Models}

The experiments were carried out in two phases first, for the diode without a sheet charge (PWB) and then, the other with insertion of sheet charge close to the potential well though, keeping all other design parameters such as intrinsic regions, size of well and doping at extrinsic regions constant in each diode.

As shown in figure 1 , these diode structures contain $\mathrm{n}^{+}$- doped regions of $A l_{0.3} \mathrm{Ga}_{0.7} A s(\mathrm{Si}), 100 \mathrm{~nm}$ long and doping concentration of $4.10 \times 10^{24} \mathrm{~m}^{-3}$ on both diode terminals (labels 1 and 4) which interact with the ohmic contacts. Labels 2 and 3 represent the left and right intrinsic regions of $A l_{0.3} G a_{0.7} A s$ with lengths $7000 \AA$ and $1500 \AA$ respectively, while a GaAs well of width $300 \AA$ was inserted between the two intrinsic regions. The experiment was done under tight control required over the thickness and composition of each epitaxial layer and performed in a RIBER V90H reactor on $n^{+}$GaAs substrates. Circular diodes with diameter $50 \mu \mathrm{m}$ were fabricated using $i$-line optical lithography in a standard wet etched process. The front and back contacts, consisting of $50 \mathrm{nmAuGe} / 13 \mathrm{nmNi} /$ $200 \mathrm{nmAu}$ were thermally evaporated and annealed providing a very low contact resistance. This was followed by an orthophosphoric based etch to produce self-aligned mesas to a depth of $1.5 \mu \mathrm{m}$ using the top contact metal as a mask. The device I-V characteristics were measured using an Agilent (keysight) B1500A Semiconductor Device Analyzer from $-3 V$ to $2 V$. The PPB diode was designed in a similar way though, with a thin sheet charge $\operatorname{doped}(\delta$-doping $)$ with Beryllium to concentration of $2.5 \times 10^{15} \mathrm{~m}^{-3}$ inserted on the left intrinsic region of diode close to the potential well to provide a good asymmetry of the two diodes. The detailed layer structure of the PWB and PPB diodes is as shown in figure 1 (a) and (b) respectively.

\section{Diodes Simulation}

The simulation of devices was carried out using two semiconductor device simulation models; the drift-diffusion (DD) and the Monte Carlo (MC) models.

\subsection{The Drift-Diffusion Model}

First, the DD model was used to obtain the $I-V$ characteristics of which some slight adjustments were made in the design parameters of diodes in order to fit the simulated results to the experiments. The left and right intrinsic regions and the well size were adjusted by some percentages within the range of $\pm 2.5 \%$. Also, a band offset of $0.25 \mathrm{eV}$ for the $\mathrm{GaAs} / \mathrm{Al}_{0.3} G a_{0.7} A s$ system was used. The simulation of the devices was carried out previously using this model and other current equations as reported in [1,
2]. The potential distributions over a mesh of 1500 points were generated in each case of the diodes by determining the doping profile for diodes using numerical approaches to solve the Poisson's equation (equation 2). Basically the models used in the simulation are as shown in the equations:

$$
\begin{gathered}
\frac{\partial J}{\partial x}=0 \\
\frac{\partial^{2} \emptyset}{\partial x^{2}}=-\rho \\
J_{n}=-q\left(\mu_{n} n E-D_{n} \frac{\partial n}{\partial x}\right)
\end{gathered}
$$

\subsection{The Monte Carlo (MC) Model}

Both the PWB and PPB diodes were simulated using MC model which allows the quantitative discussion of carrier heating effect which could not be accounted for by DD models in the previous study. The MC model has been successfully used to investigate the planar Gunn diodes [10-12] and recently the PWB diode. The model incorporates the $\Gamma-L-X$ valleys of the conduction band and also include the effect of non-parabolicity. Scattering mechanism used in the model are intervalley, acoustic and polar optical phonon scatterings. Impurity scattering is neglected in the model because the active region of diode is purely intrinsic and also, the width of potential well is smaller than the mean free path of electrons for impurity scattering to occur. A constant discretization time step which helps to determine the exact position of electron distribution at a particular time was used. This scheme allows us to track time evolution of electron distribution as well as determine the points of all sample electrons across the diode. Electron densities were calculated at each mesh point as a function of time and space coordinates. The Poisson solver was used with successive over relaxation process to obtain electric field at mesh points. A constant lattice temperature of $300 \mathrm{~K}$ was used throughout the study in both MC and DD models. The MC simulation was allowed to run over 40000 iterations at $50 \mathrm{ps}$ for each time step. The simulation also ran for $\sim 50000$ particles; transient behavior at the beginning of simulation were over within range of $100-150$ time steps. The simulation reaches stability and was averaged to give current density for every bias. The PWB structure was simulated with layers of $\operatorname{AlGaAs}(\mathrm{Si}) 0.1 \mu \mathrm{m}$ long each doped to concentration of $4.10 \times 10^{24} \mathrm{~m}^{-3}$. With the left and right intrinsic length maintained at 0.7 and $0.15 \mu \mathrm{m}$ respectively, the structure was simulated over a well thickness of $300 \AA$. The PPB diode used the same dimensions as the PWB though, with a sheet charge doped with Beryllium $(\mathrm{Be})$ atoms at a concentration of $2.50 \times 10^{15} \mathrm{~m}^{-3}$ inserted in the structure of the PPB diode as shown in Figure 1(b). The velocity of the diodes seems to be in equilibrium with the electric field though, with little sign of ballistic overshoot which will certainly impact the current especially for the PWB diode. 


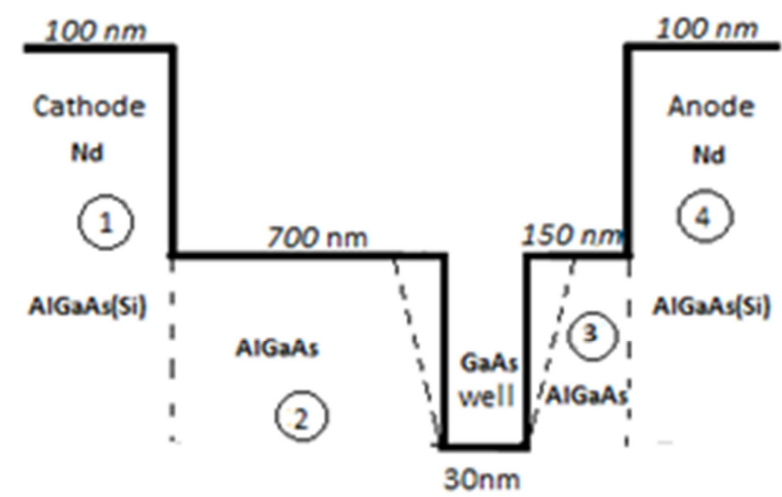

(a)

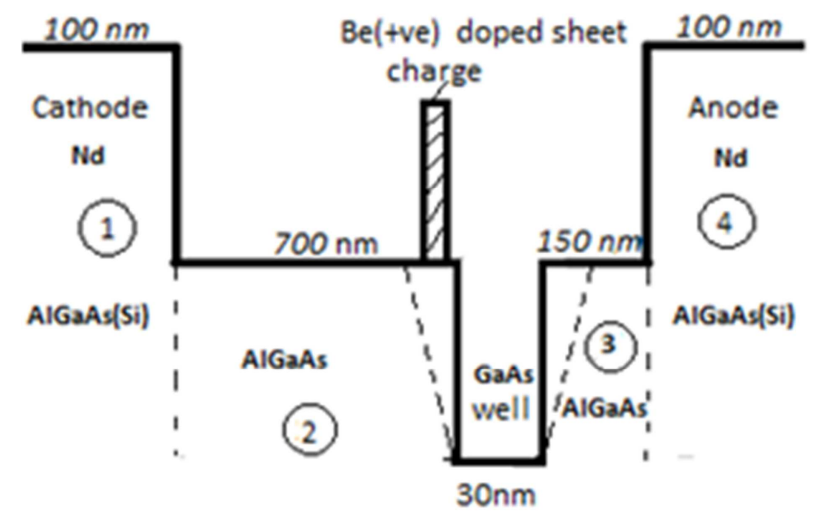

(b)

Figure 1. Heterostructures showing the design parameters of (a) PWB diode (b) hybrid PPB diode. With doped $n^{+}$regions $\left(4.10 \times 10^{24} \mathrm{~m}^{-3}\right)$, and intrinsic regions of $7000 \AA$ and $1500 \AA$ in the left and right regions respectively sandwiched between the two $n^{+}$-doped regions, and a GaAs well of $300 A$ inserted within the $i$-regions. The structure of $P B B$ diode $(1 b)$ also shows a $\delta$-doped sheet charge inserted close to the potential well.

\section{Result and Discussions}

\subsection{Comparison of the I-V Characteristics of the PWB and PPB Diodes at $1 \mathrm{~V}$}

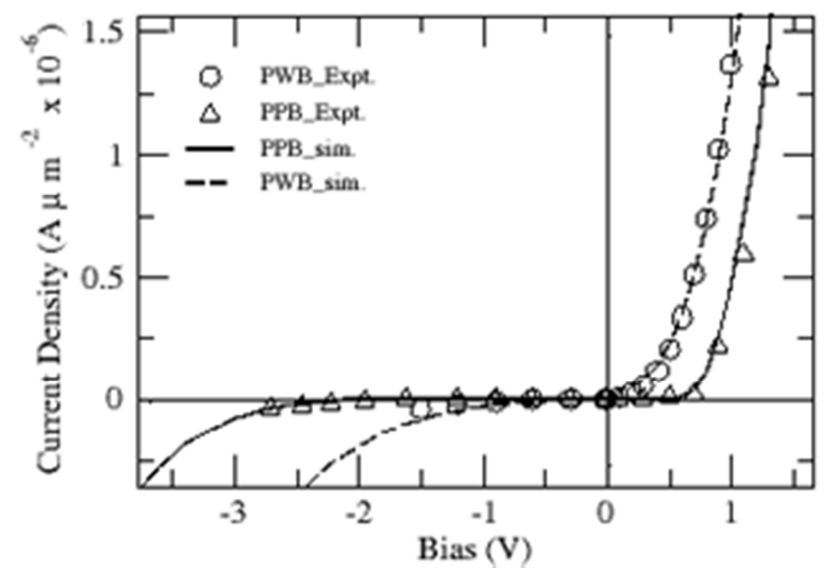

Figure 2. Current densities of the experimental and the simulated results for both the PWB and PPB diodes: circles and broken lines represent the experimental and simulated results of the $P W B$ diode respectively while triangles and solid lines represent the experimental and simulated results of the PPB diode respectively.
As shown in Figure 2 is the measured (experimental) and simulated $I-V$ characteristics of both the PWB and the PPB diodes. Measurements of devices were carried out on-wafer using a probe station at $300 \mathrm{~K}$. The $I-V$ characteristics was measured using an Agilent (Keysight) B1500A Semiconductor Device Analyzer. The results show excellent agreement between the simulation and experiments as shown in the normalized current densities in Figure 2. There is a steady rise in current without any distortion, and the current flow over the barrier is mainly by thermionic emission. The PWB and PPB diodes show an asymmetry in voltage of approximately $0.7: 2.7$ and $1: 4$ respectively at current density of $\left(0.5 \times 10^{-6} \mathrm{A \mu m}^{-2}\right)$ which verifies the diode's performance. This degree of asymmetry in a diode of this type, reflects the asymmetry of the right and left intrinsic regions. The asymmetry also shows that in the forward bias, the PWB diode gives a better performance compared to the PPB diode at same current density. If turn-on voltage of diode is defined as the voltage where the forward bias exceeds $0.1 \mu \mathrm{A \mu m} \mathrm{m}^{-2}$ then, the PWB diode turns on at a voltage of $0.33 \mathrm{~V}$ while the PPB diode turns on at a voltage of $0.93 \mathrm{~V}$. The difference in the turn-on voltage of the diodes is as a result of the $\delta$-doped sheet charge at a position close to the potential well in the PPB diode. This means that the turn-on voltage in the PPB diode is due to the sum of the charge in potential well and the $\delta$ - sheet doping. The PPB diode has however demonstrated a greater improvement in reverse bias behavior as expected compared to the PWB diode.

\subsection{Comparison of the Electric Field of the PWB and PPB Diodes at $1 \mathrm{~V}$}

Figure 3 shows the electric field distribution of the PWB and PPB diodes. The field distribution of the PWB diode rises faster at the left intrinsic region compared to the PPB diode which remains almost constant in that region. At a position of $0.4 \mu \mathrm{m}$, the field increases steadily up to $1.05 \mathrm{KV} / \mathrm{cm}$ at a position of $1 \mu \mathrm{m}$. There is abrupt rise in the electric field of the two structures with that of the PPB diode rising faster at the right intrinsic region. This means the rise in the electric field in the PPB diode is obviously because of the additional charge from the depleted sheet charge inserted in the PPB diode. In the PWB diode however, mobile electrons are very active and may create non-local and hot-carrier effect which tends to dominate the device at the left intrinsic region. Whereas the sudden rise in the electric field of the PWB diode is due to energy acquired by electrons in the potential well, the rise in the electric field of the PPB diode is due to the electrons in the depleted sheet charge and those in the well thus, a higher field is expected at the right intrinsic region of the PPB diode than it is in the PWB diode. 


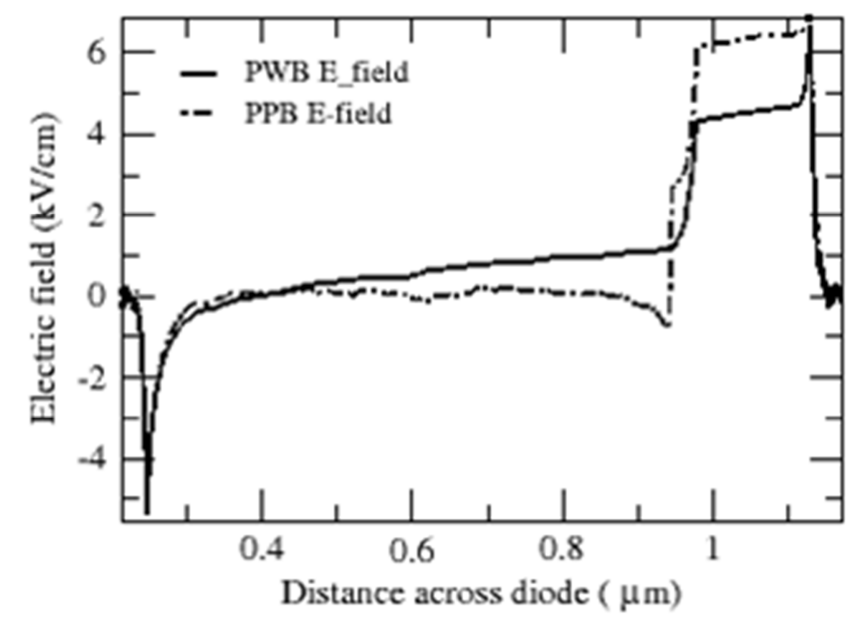

Figure 3. Comparison of the electric field of the PWB and PPB across the diodes. The field rises steadily in the PWB diode at the left intrinsic region while in the $P P B$ diode, the field remains almost constant until reaches position of the sheet charge and well.

\subsection{Comparison of the Electron Velocity at $1 \mathrm{~V}$}

The velocity of the diodes seems to be in equilibrium with the electric field though, with little sign of ballistic overshoot which will certainly impact the current density especially for the PWB diode. Due to steady rise of the electric field in the left intrinsic region of the diode, more electrons become active and this increases the average speed of the electrons in the PWB diode. This is the reason for the high mean velocity in the PWB diode. The velocity of the PWB diode reaches a value of $1.3 \times 10^{8} \mathrm{~cm} / \mathrm{s}^{2}$ at $0.65 \mu \mathrm{m}$ along the left intrinsic region at some point where the velocity of the PPB diode just begin to rise. The low velocity in the PPB diode could also be as a result of the electron-hole pair since the positively charged sheet depletes completely and this tends to reduce the electron drift thus, lowering the mobility of electrons in the PPB diode.

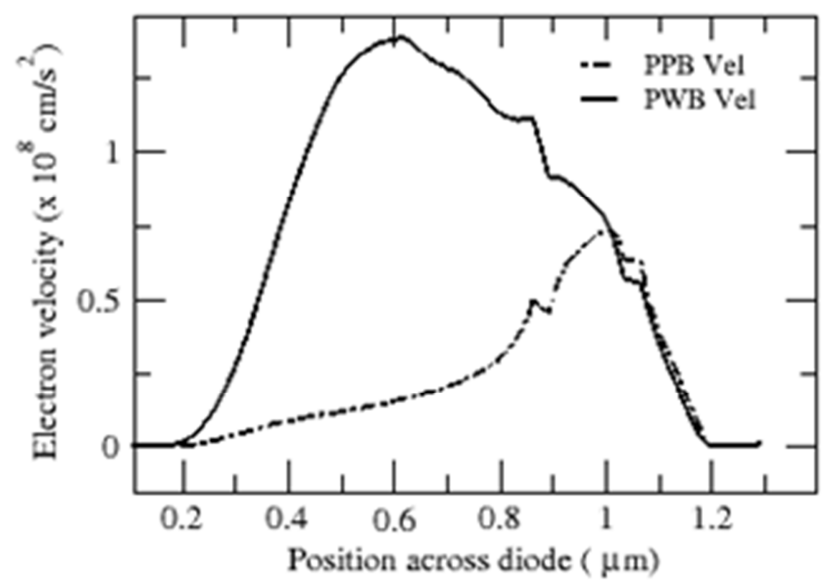

Figure 4. Comparison of the velocity of $P W B$ and hybrid PPB diode. The velocity of the $P W B$ diode rises faster with steep slope along the gamma valley and even saturates before the velocity of the $P P B$ diode reaches peak just at the position of the well.

\subsection{Behavior of Electron Energy on Diode Structures at $1 \mathrm{~V}$}

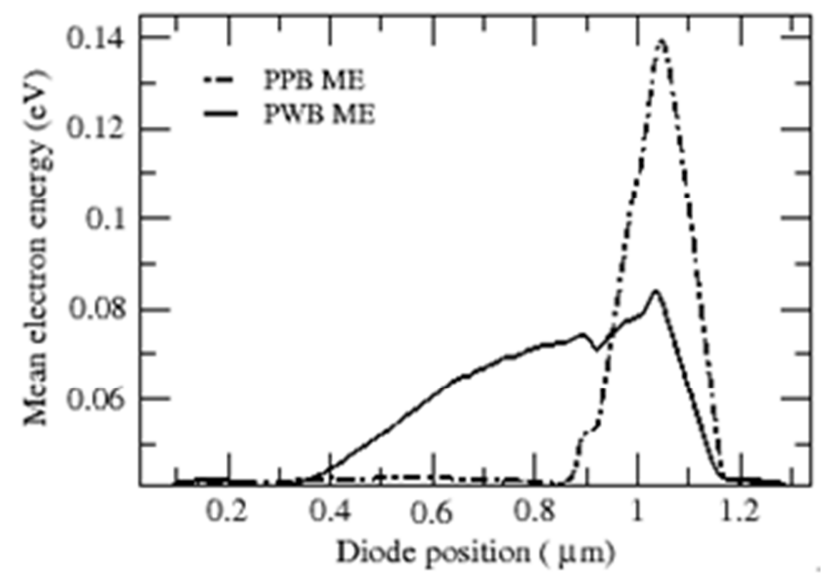

Figure 5. Average energy of the electrons in a $P P B$ and $P W B$ diodes. There is a constant energy at the left intrinsic region of the $P P B$ diode as a result of the constant field while in the PWB diode, the electron energy rises as expected.

Electron energy is a function of the velocities of the electrons. As shown in Figure 5, the mean energy of the PWB diode increases steadily from $0.3 \mu \mathrm{m}$ along the left intrinsic region and reaches a peak at $1.0 \mu \mathrm{m}$ with a value of $0.08 \mathrm{eV}$.

The reason for the steady nature of curve in the PWB along the intrinsic region is because there seems to be no electron-hole pair that would reduce the number of active electrons in the device thus, there is higher speed and collision that results to steady increase in the kinetic energy of the electrons. In the PPB diode fewer electrons are observed at the left intrinsic region due to electron-hole pair combination and so there is less collision and hence, lower kinetic energy and almost constant in the PPB diode in the left region. At position of $1 \mu \mathrm{m}$ in the PPB diode however, the mean kinetic energy abruptly increases up to $0.92 \mathrm{eV}$, due to the high density of electrons in the sheet charge and the potential well. The rate of collision increases thus, increasing the average kinetic energy of electrons.

\subsection{Behavior of Electron Densities for Each of the Diode Structures at $1 \mathrm{~V}$}

As shown in Figure 6, electron densities of the two structures were compared and it was observed that the heterojunction of the PPB diode is higher than the PWB diode and as such would offer a higher barrier. This means that the PWB diode will turn on faster than the PPB diode. The barrier height formed in the PWB diode is because of the electrons in the potential well only, while the barrier height formed in the PPB diode is due to the electrons of the depleted sheet charge and those in the well; thus forming a bigger barrier compared to the PWB diodes operating under the same conditions. As shown in figure 4, the PPB diode has a bigger barrier compared to the PWB diode though with much greater improvement in the reverse diode operation as a large current could be drawn without diode breakdown. 


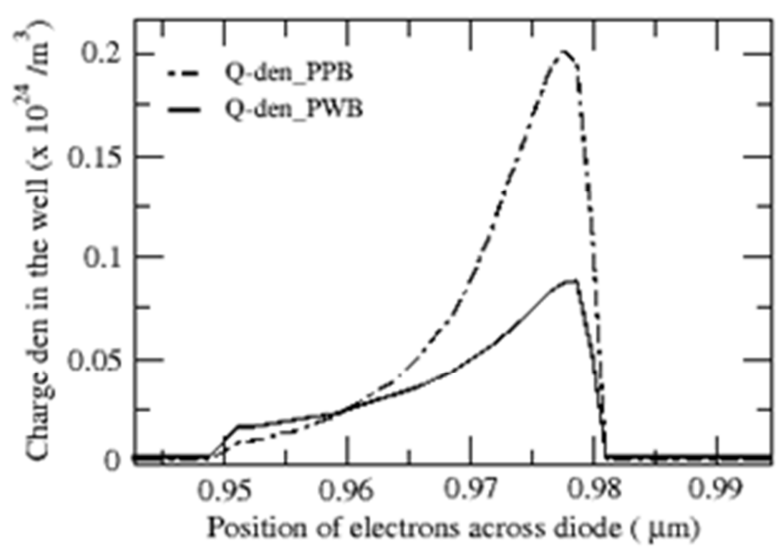

Figure 6. Shows the charge density along the diodes active region. The charge density of the PWB is due to the charge in the well only while the density of the charge in the PPB is due to the depleted electrons in the sheet charge and the charge in the well; thus has a higher barrier.

\section{DC Characteristics and Performance Analysis}

\subsection{Characteristics}

The PWBs and PPBs are similar in design to the PDBs and could also be optimized for high frequency applications in heterodyne detectors and mixers as already been demonstrated in the GaAs PDB diode. [13, 14]. The capacitance of the diodes were very low just like that of the PDB diodes and was measured to be below $1 p F$. Though the exact on-wafer measurement of the capacitance of diodes below $1 p F$ were extremely difficult for such vertical structures of this kind. Thus, to a good and reasonable approximation, the junction capacitance, $C_{j}$ was obtained using the formula $C_{j} \cong A \varepsilon / L$ where $L$ is the length of the active region of diode (active region is the region between the two doped layers of $\operatorname{AlGaAs}(S i)) ; A$, is the area of the diode and in this case the entire area of device. The capacitance of the diode was obtained to be $\approx 0.24 p F$ with diameter of diode $50 \mu \mathrm{m}$. Some important dc characteristics such as the curvature coefficient, voltage responsivity and junction resistance were also compared. The curve fitting parameters to the ideal diode equation for both diodes were estimated. The PWB diode series resistance was $15 \Omega$, while the saturation current was estimated as $5 \mu \mathrm{A}$ and ideality factor 3.3. For the PPB diode, a series resistance of $14 \Omega$ was obtained and saturation current of $16.3 \mu \mathrm{A}$ with improved ideality factor of 1.82 compared to the PWB diode with ideality factor of 3.3 [1]. Comparison was made of the curvature coefficient $\gamma=\left(d^{2} I / d V^{2}\right) /(d I / d V)$, responsivity [15] and other de quantities and results tabulated as shown in Table 1.

Table 1. Comparison of the dc quantities in a PWB and PPB diodes.

\begin{tabular}{lll}
\hline DC quantity & PWB diode & PPB diode \\
\hline Reverse saturation Current $I_{S}$ & $5 \mu \mathrm{A}$ & $16.3 \mu \mathrm{A}$ \\
Series resistance $R_{s}$ & $15 \Omega$ & $14 \Omega$ \\
Ideality factor & 3.3 & 1.82 \\
Junction capacitance $C_{j}$ & $0.23 \mathrm{pF}$ & $0.24 \mathrm{pF}$ \\
Responsivity & $6400 \mathrm{~V} / W[1]$ & $10900 \mathrm{~V} / \mathrm{W}[2]$ \\
Curvature coefficient $\gamma$ & $14.6 \mathrm{~V}^{-1}$ & $21.2 \mathrm{~V}^{-1}[2]$ \\
\hline
\end{tabular}

The estimated curvature coefficients of the PWB and PPB diode though less than the ideal Schottky barrier curvature coefficient $\left(q / K T \approx 38.6 V^{-1}\right)$, compare well with Si-based microwave detectors with curvatures of $23.2 \mathrm{~V}^{-1}$ [16] and GaN Heterostructure barrier diodes with curvature of $30 \mathrm{~V}^{-1}[5]$.

\subsection{Performance Analysis}

To better understand the performance of these diodes, the simulation of the PDB diode was included and a direct comparison with the PWB and PPB diodes as shown in Figure 7. The PDB diode was simulated using an equivalent charge of $2.5 \times 10^{15} \mathrm{~m}^{-3}$ as is in the PPB while the potential well in the PWB and PPB diodes was maintained at $30 \mathrm{~nm}$. In all diode structures, the left and right intrinsic regions of diodes were used as in the experimental design. The gradient of the log of the current density of the PDB diode was observed to rise linearly with the voltage in both the forward and reverse bias for all applied voltages. For the PWB and PPB diode however, two distinct regions were observed in the forward bias. In region 1, the gradient of the $\log$ of current density rises linearly with the voltage at certain applied biases, $(0.05<\mathrm{V}<0.1)$ for PWB and $(0.6<\mathrm{V}<0.9)$ for PPB diode. In region 2 , the $\log$ of the current density falls with the voltage above $0.1 \mathrm{~V}$ and $0.9 \mathrm{~V}$ respectively for PWB and PPB diodes. In the reverse bias, the $\log$ of the current density continue to fall with voltage at all biases though with much better reverse bias operation in the PPB diode than the PWB and PDB diodes. The turn-on voltage of the three diodes may also be compared. Thus, if the turn-on voltage of diode is defined as the voltage for which the forward bias current reaches a value of $0.1 \mu \mathrm{Am}^{-2}$. The PWB diode responds quickly to much lower voltages compared to $\mathrm{PDB}$ and $\mathrm{PPB}$ diodes with turn-on voltage of $0.32 \mathrm{~V}$ compared to PDB and PPB diodes with turn-on voltages of $0.60 \mathrm{~V}$ and $0.93 \mathrm{~V}$ respectively. It can be conclusively emphasized here that the higher value of turn-on voltage which occur in the PPB diode is due to combined charge in the potential well and that of the sheet charge though, with greater improvement in the reverse bias operation.

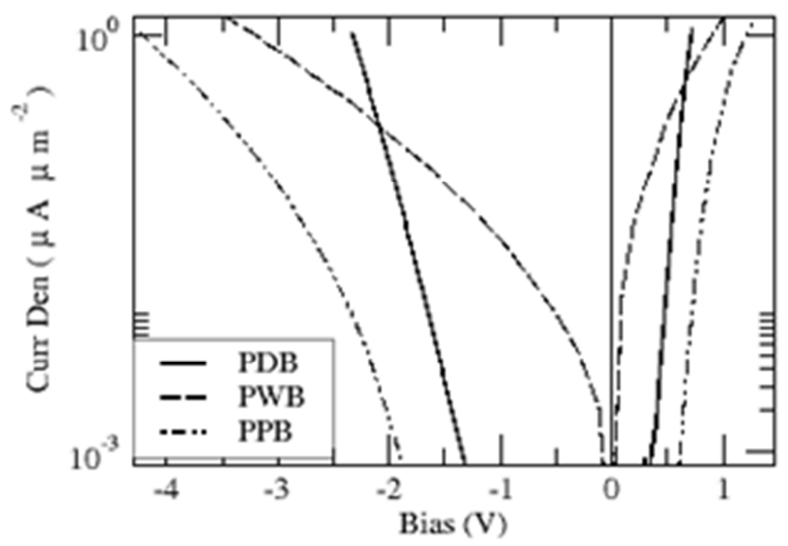

Figure 7. Shows a comparison of the I-V characteristics of $P W B, P D B$ and $P P B$ diodes. The $P P B$ diode shows a good asymmetry in the reverse bias operation though, conducts (turn-on) at high voltages compared to PWB and $P D B$ diodes in forward bias. 


\section{Conclusion}

The comparison of these two novel microwave and submillimeter-wave structures has been explored using Monte Carlo simulation model. There is good agreement between the experiments and simulations in both the PWB and PPB diode though with some adjustments within experimental uncertainties in order to achieve best fit to the $I-V$ characteristics. With very low turn-on voltage of $0.32 \mathrm{~V}$ at current density of $0.1 \mu A \mu m^{-2}$, the PWB has presented a better turn-on voltage in the forward bias compared to the PPB though, the PPB diode has offered improvement in the reverse bias action compared to both the PDB and PWB diodes. The barrier height of the PWB diode is determined by the charge in the well only while the barrier height of the PPB diode is the sum of charge in the well and the depleted sheet charge and hence, offering a higher barrier than the PWB diode. Also, it is worth-noting that the turn-on behavior of the diodes is a function of the barrier height and that is why the PWB diode has a turn-on voltage much lower than the PPB diode. With estimated curvature coefficient of 14.6 $V^{-1}$ and $21.2 V^{-1}$ for the PWB and PPB diodes respectively, this compares favorably to some extent with Si-based microwave detectors with curvatures of $23.2 \mathrm{~V}^{-1}$.

\section{Acknowledgements}

First, we would like to thank the School of Natural and Computing Sciences, University of Aberdeen for the support given to carry out this research.

We also wish to thank the lab technologists at the University of Manchester for the assistance to fabricate these diodes in their MBE and Compound Semiconductor laboratory.

\section{References}

[1] M. Akura, G. Dunn, J. Sexton and M. Missous, "Potential well barrier diodes for submillimeter wave and high frequency applications," IEEE Electron Device Lett. vol. 38, no. 4 pp. 438-440, 2017. doi:10.1109/LED.2017.2673662.J.

[2] M. Akura, G. Dunn and M. Missous, "A hybrid planar-doped potential-well barrier diode for detector applications," IEEE Trans. On Electron Dev., 642017 doi:10.1109/TED.2017.2733724.

[3] M. Akura, G. Dunn, J. Sexton and M. Missous, "GaAs/AlGaAs potential well barrier diodes: Novel diode for detector and mixer applications," Phys. Status. Solidi. A, vol. 214, pp. 17002901-7, 2017. doi:10.1002/pssa.201700290.

[4] M. Akura and G. Dunn, "Investigating the effect of temperature on barrier height of PWB diodes," Electron Lett. vol. 54, no. 1, pp. 42-43, Jan 2017. doi:10.1049/el.2017.3353.

[5] Z. Pei, A. Verma, J. Verma, H. Xing, P. Fay, D. Jena, "GaN heterostructure barrier diodes exploiting polarization induced $\delta$-doping," IEEE Electron Device Lett. vol. 35, No. 6, pp. 615-617, Jun. 2014. doi: 10.1109/LED.2014.2316140.

[6] Y. Fu, M. Mamor, M. Willander, S. Bengtsson, and L. Dillner, " $n-\mathrm{Si} / \mathrm{SiO}_{2} / \mathrm{Si}$ heterostructure barrier varactor diode design," Appl. Phys. Lett. 77, 103 (2000); doi: 10.1063/1.126891.

[7] H. Tanimoto, N. Yasuda, K. Taniguchi and C. Hamaguchi, "Monte Carlo study of hot electron transport in quantum wells," Jap. Journ. of Appl. Phys. vol. 27, no. 4, pp. 563-571, 1988.

[8] N. R. Couch and M. J. Kearney, "Hot-electron properties of GaAs planar-doped barrier diodes,” J. Appl. Phys. vol. 66, no. 10, pp. 5083-5085, 1989. doi:10.1063/1.343734.

[9] R. K. Cook, "Computer simulation of carrier transport in planar doped barrier diodes," Appl. Phys. Lett. vol. 42, no. 5, pp. 439-441, 1983. doi:10.1063/1.93963.

[10] C. Li, A. Khalid, N. Piligrim, G. Dunn and D. Cumming, "Novel planar Gunn diode operating in fundamental mode up to $158 \mathrm{GHz}$," J. of Phys: Conf. Series vol. 193, no. 1, pp 0120291-4 2009. doi: 10.1088/1742-6596/193/1/012029.

[11] T. Teoh, G. Dunn, N. Priestley, and M. Carr, "Monte Carlo modelling of multiple-transit-region Gunn diodes," Semicond. Sci. Technol. vol. 17, 1090-1095, Sept. 2002.

[12] N. Pilgrim, R. Macpherson, A. Khalid, G. Dunn and D. Cumming, "Multiple and broad frequency response Gunn diodes," Semicond. Sci. Technol. vol. 24, no. 1 pp. 105010, 2009. doi: 10.1088/0268-1242/24/10/05010.

[13] M. J. Kearney, A. Condie, and I. Dale, "GaAs planar doped barrier diodes for millimeter-wave detector application," Electron Lett., vol. 27, no. 9, pp. 721-722, Feb. 1991.

[14] R. J. Malik and S. Dixon, "A subharmonic mixer using a planar doped barrier diode with symmetric conductance," IEEE Electron Device Lett., vol. 3, no. 7, pp. 205-207, Jul. 1982.

[15] "The zero bias Schottky barrier detector diode," Agilent Technol., Santa Clara, CA, USA, Appl. Note 969.

[16] S. Y. Park, R. Yu, S. Y. Chung, P. R. Berger, P. E. Thompson and P. Fay, "Delta-doped Si/SiGe zero-bias backward diodes for micro-wave Detection", IEEE $65^{\text {th }}$ Annual Dev. Res. Conf. pp. 153 - 154, Jun. 2007. doi: 10.1109/DRC.2007.4373694. 